\title{
INSTITUTO PROFESIONAL SANTO TOMÁS. OBTENCIÓN DE LA AUTONOMÍA: UNA EXPERIENCIA CONCRETA
}

Fernando de la Jara* 


\section{RESUMEN}

Este artículo comparte algunas consideraciones $u$ observaciones sobre lo que fue para el Instituto Profesional Santo Tomás la obtención de su autonomía plena por parte del Consejo Superior de Educación (CSE), en conformidad a lo establecido en la Ley Orgánica Constitucional de Enseñanza (LOCE). Estas reflexiones están elaboradas en la perspectiva del largo plazo y no apuntan a detalles o aspectos específicos, sino más bien al proceso de verificación como un ejercicio global. Nos ha parecido importante compartir las dificultades y aportes que se experimentaron, institucionalmente, durante el proceso de verificación, así como los logros y avances que significó dicho ejercicio para el instituto.

\section{ABSTRACT}

This paper communicates some considerations and observations on what was involved in the process of obtaining full autonomous status for the Instituto Profesional Santo Tomás. Its independent status was granted by the Higher Education Council (CSE), based on the provisions established by the Constitutional Organic Law on Education (LOCE). These considerations are drafted on a long-term perspective and are not aimed at specific aspects or details. Rather, they are aimed at the process of verification as a general exercise. The authors felt it was important to share these difficulties and the input contributed ainstitutionallya during the verification procedure, as well as the achievements and the process that this experience involved for the Institute. 


\section{INSTITUTO PROFESIONAL SANTO TOMÁS. OBTENCIÓN DE LA AUTONOMÍA: UNA EXPERIENCIA CONCRETA}

Introducción

El presente trabajo tiene por objeto compartir algunas consideraciones $\mathrm{u}$ observaciones sobre lo que fue para el Instituto Profesional Santo Tomás (IPST) la obtención de su autonomía plena, por parte del Consejo Superior de Educación (CSE), en conformidad a lo establecido en la Ley Orgánica Constitucional de Enseñanza (LOCE).

Estas reflexiones fueron ya expuestas en el seminario que el Consejo organizara, en septiembre de este año, con el fin de analizar lo que ha sido su rol en el ejercicio del sistema de acreditación o verificación institucional, en conjunto con algunas de las instituciones participantes de dicho proceso.

Alcance de nuestras consideraciones

a) Nuestras reflexiones acerca de lo que significó para la IPST el proceso de verificación institucional están elaboradas en la perspectiva del largo plazo. Así, entonces, no apuntan a detalles o aspectos específicos, sino más bien al proceso de verificación como un ejercicio global.

b) Nos ha parecido importante compartir las dificultades y aportes que se experimentaron, institucionalmente, durante el proceso de verificación, así como los logros y avances que significó dicho ejercicio para el instituto.

c) Como una manera de ordenar nuestras consideraciones o evaluaciones, decidimos presentarlas agrupadas en fortalezas y debilidades. 
Fortalezas del Proceso de Verificación en el Instituto Profesional Santo Tomás.

1. Instalación de una cultura de evaluación y Verificación del Proyecto de Desarrollo Institucional

El primer efecto que se produjo en la institución, a partir de su ingreso al Sistema de Verificación del CSE, fue el de hacerse cargo de la evaluación de su Proyecto de Desarrollo Institucional de una manera orgánica y sistemática. Lo anterior se tradujo en dos acciones:

a) Socialización de proyecto. En este sentido se desarrollaron una serie de tareas y acciones tendientes a compartir el proyecto al interior de la institución. Esto no significa, en modo alguno, que con anterioridad se hubiese pretendido esconderlo, sino que ahora se toma conciencia que este es la expresión del compromiso y voluntad de ser de la institución.

b) Institucionalización de la Verificación. El ingreso al Sistema de Verificación planteó una nueva tarea para la institución, la que no estaba recogida en su estructura organizacional. Esto la obligó a crear y determinar, dentro de su estructura, las unidades y responsables que darían dar cuenta de esta acción.

\section{Redefinición del Proyecto de Desarrollo Institucional}

De alguna manera, ya está dicho que el Proyecto de Desarrollo Institucional era un documento fundacional que, aunque genuino e importante, resultaba bastante desconocido para la comunidad académica y, entonces, constituía un referente de desarrollo muy alejado y sin mayor gravitación. La socialización y discusión del proyecto, al interior de la comunidad académica, hizo manifiesta la necesidad de una redefinición y contextualización de dicho documento. Esto se materializó a través de las siguientes acciones:

a) El proyecto entendido como un "compromiso" que se debe cumplir. La discusión y evaluación del proyecto hizo tomar conciencia a los actores que estaban frente a una declaración de intenciones voluntariamente formulada, que los comprometía frente a los alumnos y profesores y que, por lo 
tanto, sus alcances debían ser cuidadosamente estudiados en función de las capacidades institucionales, a objeto de dar un contenido real al proyecto.

b) El proyecto como una "obligación" de todos. Derivado de lo anterior, la comunidad académica del instituto fue tomando conciencia de que el proyecto constituía un compromiso que los involucraba a todos. Se comenzó a entender que, en la medida en que todos lo conocieran, aceptaran y participaran de sus contenidos, se podría lograr avanzar en un desarrollo acorde a lo comprometido.

c) Contextualización del proyecto a una "realidad posible". Como resultado de lo anterior se vio la necesidad de redefinir el proyecto, en algunos de sus alcances o contenidos, a la luz de la realidad posible de la institución. Sin duda, la distancia que existía entre el momento de su formulación y el ejercicio de su evaluación, ponían de relieve algunas diferencias sustanciales entre lo que se había pensado y comprometido y la realidad del IPST, sus fines y capacidades.

3. Claridad en la estructura de gobierno y organización del trabajo

El desarrollo de los ejercicios de socialización, evaluación y contextualización del proyecto, al interior del instituto, derivó en un nuevo Programa de Desarrollo modificado, que se traducía, a su vez, en un programa de trabajo operacionalizado y por todos conocido. La formulación de estos nuevos compromisos y sus planes para cumplirlos, obligó a la institución a evaluar, adecuar y enriquecer su estructura de gobierno. Esta tarea se puede resumir en los siguientes hechos o acciones:

a) Claridad en la toma de decisiones, Organismos Colegiados y Unipersonales. Si el Proyecto de Desarrollo Institucional era un compromiso y obligación de todos, las decisiones que afectaran a su cumplimiento requerían, también, un mayor grado de participación de todos los involucrados. Se trataba de que las decisiones se tomaran, informadamente, en lo ámbitos de competencia respectivos y esto significó, en los hechos, darse una nueva institucionalidad de gobierno interior. Se crearon y reformularon los Organismos Colegiados y 
Unipersonales, en cuanto a sus composiciones, atribuciones y normas de funcionamiento. En síntesis, se ordenó el proceso de Toma de Decisiones en función del proyecto y las responsabilidades que este establecía.

b) Planes de trabajo por Unidades. Como resultado de todo este nuevo ordenamiento, cada unidad fue construyendo sus planes de trabajo a partir de una matriz general diseñada y oficializada por las diferentes unidades centrales, tanto académicas como administrativas. Dichos planes constituyeron una especie de carta de navegación para las Unidades, especialmente para las escuelas, las que, a partir del Proyecto Institucional, sabían ahora con más claridad cómo cumplirían con sus objetivos y compromisos. Sin duda esto logró mejorar la claridad de todos los actores involucrados, agrupados en unidades, respecto de sus obligaciones para con el proyecto.

c) Claridad en las evaluaciones de desempeño. El IPST, al igual que cualquier institución formal, posee una estructura con diferentes cargos, unidades y responsabilidades, adscritos a la función que cada cual desempeña. En la medida en que se fueron construyendo los programas de trabajo de cada unidad, se fueron también estableciendo, con mayor conocimiento compartido, las responsabilidades de los diferentes actores involucrados en la concreción de dichos programas. Todo esto contribuyó, al interior de la organización, a que todos tuvieran una mayor conciencia de sus obligaciones y, al mismo tiempo, a evaluar con una mayor certeza el cometido y cumplimiento de los objetivos de las diferentes unidades y sus integrantes. Lo anterior siempre en función del Proyecto de Desarrollo de la institución y, consecuentemente, de los objetivos y tareas que a cada unidad le correspondían.

d) Fortalecimiento del gobierno interior. Lo anterior se tradujo en una gestión más inteligible para toda la comunidad del instituto. Los objetivos, planes de trabajo y metas eran, ahora, conocidos y compartidos por todos. Esto, sin duda, fortaleció el gobierno interior de la institución. Las medidas que se tomaban podían ser entendidas y justificadas a la luz del proyecto, las acciones ordenadas en función de ejes conocidos, sentidos como genuinos y legítimos. El llegar a este estado de desarrollo le permitió al instituto avanzar en su consolidación 
a un mejor ritmo, importantes economías de trabajo a partir de una mejor claridad de los objetivos y, finalmente, un esfuerzo mancomunado en función de su proyecto.

e) Planificación en la adquisición de los recursos, en función del Proyecto de Desarrollo Institucional. Un hecho que no es menor en las instituciones de educación superior es el de tener claridad respecto de quien asigna las prioridades de inversión de los recursos y los criterios que se ocupan para dichas asignaciones. Cuando los criterios ocupados no son claros, explícitos y en función de los objetivos comprometidos, se producen asimetrías en el desarrollo de la institución y, finalmente, se resiente el Proyecto Institucional en su totalidad. En el Instituto Profesional Santo Tomás, el haber generado un Proyecto de Desarrollo Institucional con todas las características ya señaladas, permitió que la administración de los recursos financieros, por definición escasos, se llevara a cabo con criterios claros, extraídos de su proyecto y se priorizara a partir de las opiniones y necesidades planteadas por las diferentes unidades, y en los ámbitos de cada una de ellas.

Lo anterior cobró especial importancia en el caso de las definiciones e implementaciones de los recursos de apoyo a la docencia, la definición e implementación de las bibliotecas, y la definición y dotación de los cuadros de académicos contratados por jornada.

Debilidades del Proceso de Verificación en el Instituto Profesional Santo Tomás

A continuación planteamos una serie de debilidades experimentadas por nuestro instituto en el proceso de acreditación o verificación de su Proyecto de Desarrollo Institucional administrado por el Consejo Superior de Educación. Estas observaciones apuntan tanto a la mecánica del proceso como a sus resultados generales para el instituto.

Estamos concientes de que algunas de la observaciones formuladas obedecen a los contenidos de la LOCE, que obliga al Consejo a actuar de determinadas maneras, aunque ello no desmiente el contenido y consecuencias de las debilidades planteadas. 
a) El Cumplimiento de Acciones como eje del proceso. El proceso de acreditación o verificación transcurre, por parte de la institución, a través del camino del cumplimiento de acciones dispuesto por el Consejo. Sin duda alguna, las acciones son derivadas del Proyecto de la Institución y el Consejo sólo vela por que se cumpla lo comprometido. Nada hay en esto de antojadizo o intervencionista.

El problema se presenta porque esta forma de mirar a la institución es, en general, bastante pobre y parcial. En numerosas oportunidades el Consejo no tiene una visión amplia y correcta de los esfuerzos globales de la institución, en el logro de su proyecto. Es frecuente, también, que las acciones pierdan importancia con el transcurso del tiempo y, sin embargo, persisten como el sujeto de evaluación de la institución y su desarrollo.

Son numerosas las iniciativas que se desechan o asumen, tanto por condiciones internas como externas, lo cual cambia radicalmente las prioridades específicas o estratégicas de la institución. Esto no significa abandonar el proyecto o variarlo sustantivamente respecto de sus objetivos generales, sino, más bien, tener una flexibilidad y disposición a determinar acciones diferentes cuando la situación lo reclame. La exigencia de cumplimiento del proyecto a la letra de su formulación puede, a veces, obstaculizar acciones o planes que no estaban, inicialmente, incluidos en el mismo.

Para la entidad, todo esto resulta difícil de explicar y demostrar al Consejo, toda vez que la comunicación gira en torno a la evaluación de las acciones prescritas.

Lo anterior se puede traducir en una especie de incentivo perverso para las instituciones, en el sentido que proyectos más reducidos y escuálidos tienen una mayor posibilidad de lograr su plena autonomía.

b) La continuidad en el tiempo. La mecánica de la acreditación o verificación del Proyecto de Desarrollo Institucional está destinada a perder continuidad y velocidad en el tiempo.

Los ritmos que implican las visitas de verificación, la generación de los informes de las comisiones, la respuesta de la institución y el acuerdo respectivo del Consejo, demandan un tiempo no menor que, inevitablemente, resulta más lento que el dina- 
mismo de la institución y sus necesidades de una pronta retroalimentación por parte del Consejo.

Es por este motivo que, como señalamos, en la letra anterior, quede la evaluación de acciones, a veces, como algo poco relevante para la institución y, lo que es más grave, de importancia no compartida en la evaluación del proyecto que hace la institución y el Consejo.

c) Proceso mediatizado por los "pares evaluadores". Como todos sabemos, el Consejo administra el proceso de verificación, en parte, a partir de los informes que le entregan los pares evaluadores contratados para estos efectos. Cabe destacar que siempre el Consejo ha dispuesto y contado con un equipo de indiscutible calidad y capacidad, tanto para evaluar a las instituciones como a sus quehaceres específicos, ya sean estos generados en el ámbito de las carreras, la extensión y/o la investigación.

El problema que acarrea esta mecánica de evaluar a las instituciones, es que ellas no se sienten conocidas y evaluadas realmente por el Consejo, sino por personas ajenas al proceso, que no siempre informan con claridad y amplitud a los consejeros. Este sentimiento de las instituciones guarda relación con el hecho de que la mirada de los pares evaluadores se enfoca a las acciones dispuestas por el Consejo y no a lo que las instituciones desean mostrar o compartir y a que, en muchas oportunidades, dichos pares son personas que no participan del proceso en su totalidad a lo largo del tiempo.

d) Conformación de las comisiones de pares evaluadores. Como se señalara en la letra anterior, las comisiones de pares evaluadores varían en el tiempo respecto de sus integrantes para una misma institución. Esto muchas veces se traduce en una pérdida de continuidad en el traspaso de la información que la institución hace al par evaluador. Es imposible que los documentos oficiales, que operan entre la institución y el Consejo, contengan toda la información detallada que la institución ha compartido con sus evaluadores en las visitas acumuladas en el tiempo. Así, entonces, los cambios de pares evaluadores implican, a veces, cambios en los puntos de interés y énfasis puestos en los diferentes aspectos de la evaluación. Esto produce el sentimiento en las instituciones de que el 
Consejo no las conoce y las evalúa por referencias de terceros, con los inconvenientes que esto conlleva.

e) La Examinación. Como un proceso paralelo al de Verificación del cumplimiento del proyecto, se desarrolla en las instituciones el de Examinación. Sin duda alguna, este ejercicio constituye un dato duro respecto del avance y progresión de los alumnos. En cualquiera de sus modalidades, se lleva a cabo sin mayores problemas administrativos para ambas partes. Sus resultados constituyen evaluaciones de gran contenido para las instituciones y sus sistemas de control sobre el ejercicio de la docencia impartida. En general, los resultados de este proceso, informados por el Consejo, están fundados en resultados inapelables que las instituciones asumen y ocupan en su gestión.

El único problema que se experimenta con la Examinación es el del tiempo que transcurre entre el ejercicio y los informes o resultados del mismo. Muchas veces este tiempo puede durar un año calendario y a veces un poco más. Esto produce una imposibilidad práctica de tomar medidas acorde a las requeridas de acuerdo a los resultados experimentados. Además, las instituciones comienzan, paulatinamente, a ver este proceso como algo distante y separado, que no forma parte del proceso global de evaluación del cumplimiento de su proyecto. 